IFUP-TH/2002-25

\title{
NON-ABELIAN MAGNETIC MONOPOLES AND DYNAMICS OF CONFINEMENT
}

\author{
Stefano BOLOGNESI \\ Scuola Normale Superiore - Pisa \\ Istituto Nazionale di Fisica Nucleare - Sezione di Pisa \\ Piazza dei Cavalieri 7, Pisa, Italy
}

Kenichi KONISHI

Dipartimento di Fisica "E. Fermi" - Università di Pisa

Istituto Nazionale di Fisica Nucleare - Sezione di Pisa

Via Buonarroti, 2, Ed. C, 56127 Pisa, Italy

konishi@df.unipi.it

\begin{abstract}
:
Magnetic monopoles having non-Abelian charges have been found recently to play a crucial role in the infrared in a class of supersymmetric gauge theories. We argue that these "dual quarks" can naturally be identified with the non-Abelian magnetic monopoles of the type first discussed by Goddard, Nuyts and Olive. Our argument is based on a few simple observations as regards to their charge structure, flavor quantum numbers, and some general properties of electromagnetic duality.
\end{abstract}




\section{Introduction}

With the advent of the exact Seiberg-Witten solution in $\mathcal{N}=2$ supersymmetric gauge theories [1, 2, 3, our understanding of nonperturbative dynamics of non-Abelian gauge theories received a significant boost. In particular, the behaviors of topologically nontrivial excitations such as magnetic monopoles or dyons, which were earlier known mainly from semi-classical analyses, can now be studied exactly in wide classes of models. For instance, many examples of strongly interacting

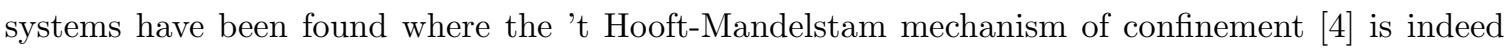
realized.

However, a series of studies in a wider class of softly broken $\mathcal{N}=2$ supersymmetric gauge theories have shown [5, [6] that actually much richer varieties of dynamical possibilities exist. Confining vacua in $S U\left(n_{c}\right), U S p\left(2 n_{c}\right)$ or $S O\left(n_{c}\right)$ gauge theories with softly broken $\mathcal{N}=2$ supersymmetry, with various number of flavors $n_{f}<2 n_{c}, 2 n_{c}+2, n_{c}-2$, respectively, have been found to fall, roughly speaking, into the following three categories [6]:

(i) In some of the vacua (the $r=0$ or $r=1$ vacua of $S U\left(n_{c}\right)$ theories; also confining vacua of all flavorless cases), the gauge group of the low-energy dual theory is the maximal Abelian subgroup $U(1)^{R}$, where $R$ is the rank of the original gauge group; confinement is described by 't Hooft-Mandelstam mechanism;

(ii) In the general $r$ vacua $\left(2 \leq r<\frac{n_{f}}{2}\right)$ of the $S U\left(n_{c}\right)$ theory, the effective low-energy theory is a non-Abelian $S U(r) \times U(1)^{n-r}$ gauge theory; among the massless degrees of freedom are the magnetic monopoles in the fundamental representation of dual $S U(r)$ gauge group. Their condensation, together with that of Abelian monopoles of the $U(1)^{n-r}$ factors, describes the confinement of the original electric theory as a generalized dual Meissner effect. The vacua in the same universality classes appear in $U S p\left(2 n_{c}\right)$ and $S O\left(n_{c}\right)$ theories with nonzero bare hypermultiplet (quark) masses;

(iii) In the $r=\frac{n_{f}}{2}$ vacua of $S U\left(n_{c}\right)$ theory, as well as in all of the confining vacua of $U S p\left(2 n_{c}\right)$ and $S O\left(n_{c}\right)$ theories with vanishing bare quark masses 1 , the low-energy degrees of freedom involve relatively non-local states: the effective theory is a deformed superconformal theory, i.e., near an infrared fixed-point. There is no local Lagrangian description of such vacua.

The aim of this paper is to argue that the "dual quarks" appearing in the $r$-vacua $(r \geq 2)$ of the softly broken $\mathcal{N}=2 S U\left(n_{c}\right)$ theories, (ii) above, are nothing but the non-Abelian magnetic monopoles of the type first discussed by Goddard, Nuyts and Olive [7] and studied extensively by E. Weinberg [8]. Our argument is based on the simple observations as regards to their charges, flavor quantum numbers, and some general properties of electromagnetic duality.

\section{Semiclassical Non-Abelian Monopoles}

Consider a gauge theory where the gauge group $G$ is broken,

$$
G \stackrel{\langle\phi\rangle \neq 0}{\Longrightarrow} H
$$

\footnotetext{
1 There are exceptions to this rule for small values of $n_{f}$ and $n_{c}$, e.g., $U S p(2)=S U(2)$ case. See the footnote 18 of $[6]$.
} 
where the unbroken group $H$ is in general non-Abelian. In order to have a nontrivial finite-energy configuration, the scalar fields and gauge field must behave asymptotically as

$$
\mathcal{D} \phi \stackrel{r \rightarrow \infty}{\longrightarrow} 0 \Rightarrow \phi \sim U \cdot\langle\phi\rangle \cdot U^{-1}, \quad A_{i}^{a} \sim U \cdot \partial_{i} U^{\dagger} \rightarrow \epsilon_{a i j} \frac{r_{j}}{r^{3}} G(r),
$$

representing nontrivial elements of $\Pi_{2}(G / H)=\Pi_{1}(H)$. The function $G(r)$ can be chosen as

$$
G(r)=\beta_{i} T_{i}, \quad T_{i} \in \text { Cartan Subalgebra of } H .
$$

Topological quantization leads to the result [7] that the "charges" $\beta_{i}$ take values which are weight vectors of the group $\tilde{H}$ where $\tilde{H}=$ dual of $H$. The dual of a group (whose roots vectors are $\alpha$ 's) is defined by the root vectors which span the dual lattice, i.e., $\tilde{\alpha}=\alpha / \alpha^{2}$. Examples of pairs of the duals are:

\begin{tabular}{ccc}
\hline$S U(N) / Z_{N}$ & $\Leftrightarrow$ & $S U(N)$ \\
$S O(2 N)$ & $\Leftrightarrow$ & $S O(2 N)$ \\
$S O(2 N+1)$ & $\Leftrightarrow$ & $U S p(2 N)$ \\
\hline
\end{tabular}

Table 1: Some examples of dual pairs of groups

To be concrete we consider a general (supersymmetric or non supersymmetric) $S U\left(n_{c}\right)$ gauge theory with an appropriate set of scalar fields in the adjoint representation. As will be mentioned at the end, our analysis applies equally well to other gauge groups. We assume that the minimum of the potential is such that the gauge group is broken spontaneously as

$$
S U\left(n_{c}\right) \rightarrow S U(r) \times U(1)^{n_{c}-r} .
$$

For instance the VEV of a scalar can be taken in the diagonal form

$$
\langle\phi\rangle=\left(\begin{array}{cccc}
v_{0} \mathbf{1}_{r \times r} & \mathbf{0} & \ldots & \mathbf{0} \\
\mathbf{0} & v_{r+1} & \ldots & 0 \\
\vdots & \vdots & \ddots & \vdots \\
\mathbf{0} & 0 & \ldots & v_{n_{c}}
\end{array}\right), \quad r v_{0}+\sum_{j=r+1}^{n_{c}} v_{j}=0,
$$

where $v_{i}$ 's are all different. Let us write the asymptotic Higgs field more compactly as

$$
\phi_{0}=\mathbf{h} \cdot \mathbf{H}
$$

where the $n_{c}-1$ rank vector $\mathbf{h}$ describes the scalar VEV, while $\mathbf{H}$ represents the generators in the Cartan subalgebra of $S U\left(n_{c}\right)$. If $\mathbf{h}$ had non-zero inner products with all of the root vectors of $S U\left(n_{c}\right)$ then the gauge group would be maximally broken to $U(1)^{n_{c}-1}$ group and Abelian monopoles having respective $U(1)$ charges would appear. We have nothing to add about such a system.

Here we consider the case in which $\mathbf{h}$ is orthogonal to the root vectors of a $S U(r)$ subgroup. The simplest way to detect the presence of the non-Abelian monopoles is to consider various $S U(2)$ subgroups generated by

$$
t_{1}=\frac{1}{\sqrt{2 \alpha^{2}}}\left(E_{\alpha}+E_{-\alpha}\right) ; \quad t_{2}=-\frac{i}{\sqrt{2 \alpha^{2}}}\left(E_{\alpha}-E_{-\alpha}\right) ; \quad t_{3}=\alpha^{*} \cdot \mathbf{H},
$$

where $\alpha$ is a root vector associated with broken generators $E_{ \pm \alpha}$ and $\alpha^{*} \equiv \alpha / \alpha^{2}$. In particular we consider those $S U(2)$ groups which do not commute with the unbroken subgroup $S U(r)$. In 
the notation of Eq. 2.4 these correspond to $S U(2)$ subgroups acting in the $[i-k]$ subspaces, where $i=1,2, \ldots, r$, and $k=r+1, r+2, \ldots n_{c}$. The symmetry breaking (2.3) induces the Higgs mechanism in such an $S U(2)$ subgroup,

$$
S U(2) \Longrightarrow U(1) .
$$

By embedding the known 't Hooft-Polyakov monopole [9] lying in this subgroup, and adding a constant term for $\phi$ so that it behaves correctly asymptotically, one easily constructs a solution of the $S U\left(n_{c}\right)$ equation of motion (see E. Weinberg [8]):

$$
A_{i}(\mathbf{r})=A_{i}^{a}(\mathbf{r}, \mathbf{h} \cdot \alpha) t_{a} ; \quad \phi(\mathbf{r})=\chi^{a}(\mathbf{r}, \mathbf{h} \cdot \alpha) t_{a}+\left(\mathbf{h}-(\mathbf{h} \cdot \alpha) \alpha^{*}\right) \cdot \mathbf{H},
$$

where

$$
A_{i}^{a}(\mathbf{r})=\epsilon_{a i j} \frac{r^{j}}{r^{2}} A(r) ; \quad \chi^{a}(\mathbf{r})=\frac{r^{a}}{r} \chi(r), \quad \chi(\infty)=\mathbf{h} \cdot \alpha
$$

is the standard 't Hooft-Polyakov solution. Note that $\phi(\mathbf{r}=(0,0, \infty))=\phi_{0}$.

The mass of this monopole for the minimum magnetic charge is given by the standard formula (we assume the BPS situation)

$$
M=\frac{4 \pi}{g} \mathbf{h} \cdot \alpha=\frac{4 \pi}{g}\left|v_{0}-v_{k}\right| .
$$

By an appropriate field redefinition $v_{0}$ can be always taken to be positive. Also, for generic, unequal values of $v_{i}$, it is possible, by using a Weyl transformation, to take the scalar VEV so that

$$
\left|v_{0}-v_{r+1}\right|<\left|v_{0}-v_{k}\right|, \quad k=r+2, r+3, \ldots, n_{c} .
$$

By considering various $S U(2)$ subgroups acting on $[i, r+1]$ subspaces, where $i=1,2, \ldots, r$, we find that there are precisely $r$ degenerate solutions with the same mimimum mass,

$$
M=\frac{4 \pi}{g}\left|v_{0}-v_{r+1}\right| \text {. }
$$

They are transformed to each other by the Weyl transformations. By construction these solutions carry also a unit (magnetic) charge with respect to the $U_{0}(1)$ gauge group, which is generated by

$$
Q_{0}=\left(\begin{array}{cccc}
\frac{1}{r} \mathbf{1} & 0 & \ldots & \ldots \\
0 & -1 & 0 & \ldots \\
\vdots & 0 & 0 & \ldots \\
\vdots & 0 & \ldots & \ddots
\end{array}\right)
$$

The system, furthermore, has $n_{c}-r-1$ Abelian monopoles, each with the minimal charge in

$$
\operatorname{Diag} Q_{\ell}=[\underbrace{0,0, \ldots, 0}_{\ell}, 1,-1,0, \ldots, 0], \quad r \leq \ell \leq n_{c}-1
$$

and with mass

$$
M_{\ell}=\frac{4 \pi}{g}\left|v_{\ell}-v_{\ell+1}\right| .
$$

For appropriate choice of the scalar vacuum expectation values (VEVS) (and arranging them appropriately by Weyl transformations) there are thus an $r$-plet of "non-Abelian" monopoles and $n_{c}-r-1$ Abelian monopoles with minimum charges and minimum masses that are stable . 


\begin{tabular}{ccccccc} 
Monopoles & $S U(r)$ & $U_{0}(1)$ & $U_{1}(1)$ & $U_{2}(1)$ & $\ldots$ & $U_{n_{c}-r-1}(1)$ \\
\hline$q$ & $\underline{r}$ & 1 & 0 & 0 & $\ldots$ & 0 \\
\hline$e_{1}$ & $\underline{1}$ & 0 & 1 & 0 & $\ldots$ & 0 \\
\hline$e_{2}$ & $\underline{1}$ & 0 & 0 & 1 & $\ldots$ & 0 \\
\hline$\vdots$ & $\vdots$ & $\vdots$ & $\vdots$ & $\vdots$ & $\ddots$ & $\vdots$ \\
\hline$e_{n_{c}-r-1}$ & $\underline{1}$ & 0 & 0 & 0 & $\ldots$ & 1 \\
\hline
\end{tabular}

Table 2: Stable magnetic monopoles of minimum masses and their charges

We are going to argue that the degenerate $r$-plet of monopoles can, under appropriate conditions, emerge as a multiplet in the fundamental representation of the dual $S U(r)$ group. Their quantum numbers, together with those of Abelian monopoles, would appear as in Table 2.

As an illustration of the above construction, consider the simplest nonotrivial case with $n_{c}=$ $3, r=2$, i.e., an $S U(3)$ theory with symmetry breaking,

$$
S U(3) \stackrel{\langle\phi\rangle}{\longrightarrow} S U(2) \times U(1), \quad\langle\phi\rangle=\left(\begin{array}{ccc}
v & 0 & 0 \\
0 & v & 0 \\
0 & 0 & -2 v
\end{array}\right) .
$$

By considering a broken $S U(2)$ subgroup (" $U$ "-spin),

$$
t^{4}=\frac{1}{2}\left(\begin{array}{ccc}
0 & 0 & 1 \\
0 & 0 & 0 \\
1 & 0 & 0
\end{array}\right) ; \quad t^{5}=\frac{1}{2}\left(\begin{array}{ccc}
0 & 0 & -i \\
0 & 0 & 0 \\
i & 0 & 0
\end{array}\right) ; \quad \frac{t^{3}+\sqrt{3} t^{8}}{2}=\frac{1}{2}\left(\begin{array}{ccc}
1 & 0 & 0 \\
0 & 0 & 0 \\
0 & 0 & -1
\end{array}\right)
$$

one finds a solution

$$
\begin{aligned}
& \phi(\mathbf{r})=\left(\begin{array}{ccc}
-\frac{1}{2} v & 0 & 0 \\
0 & v & 0 \\
0 & 0 & -\frac{1}{2} v
\end{array}\right)+3 v\left(t_{4}, t_{5}, \frac{t_{3}}{2}+\frac{\sqrt{3} t_{8}}{2}\right) \cdot \hat{r} \phi(r), \\
& \vec{A}(\mathbf{r})=\left(t_{4}, t_{5}, \frac{t_{3}}{2}+\frac{\sqrt{3} t_{8}}{2}\right) \wedge \hat{r} A(r),
\end{aligned}
$$

where $\phi(r)$ and $A(r)$ are 't Hooft's functions with $\phi(\infty)=1, \phi(0)=0, A(\infty)=-1 / r$. A second solution with the same energy can be constructed by using another $S U(2)$ group ("V"-spin) acting in the [2-3] subspace. Together, they form a doublet of the unbroken (dual) $S U(2)$ group. They carry a unit $t^{8}$ charge. There are no other "Abelian" monopoles.

Existence of the magnetic monopoles transforming as in the fundamental representation of the dual $S U(r)$ group, might appear to be in contradiction with some earlier results on the non-existence of dyons with non-Abelian charges [10], but it is not. Our monopoles carry nonzero charges with respect to $S U(r)$ as well as to one of the $U(1)$ factors, but both refer to the dual of the original subgroups. The arguments excluding the possibility of "colored dyons" (referring to the GUTs magnetic monopoles carrying the electric, color $S U(3)$ quantum numbers) do not apply. The crucial point is that the monopoles with non-Abelian charges (2.8), are to transform linearly under the dual of the unbroken gauge group, and not under the original unbroken subgroup.

Note that in the presence of an unbroken, asymptotically-free gauge interactions, the question of the validity of semiclassical approximation sometimes used in these discussions, is a subtle one. 
For instance, it is not justified to smoothly approach the limit of non-maximal breaking such as in (2.3), starting from the maximally broken case, by letting some of the diagonal elements of the scalar VEVS to coincide. Some of the Abelian monopoles would become apparently massless and spatially infinitely extended in such a limit; any result involving these light states lies however beyond the scope of the semi-classical approximation. In fact, it would hardly make any sense to consider the spontaneously broken $S U(2) \stackrel{v}{\longrightarrow} U(1)$ theory and to take the limit $v \rightarrow 0$ to attempt to find out what happens to the 't Hooft - Polyakov monopole, with a semi-classical method!

For the same reasons the system characterized by the scalar VEVS, Eq.(2.4), is never really semiclassical, even if

$$
v_{0} \gg \Lambda ; \quad v_{j} \gg \Lambda, \quad \forall j
$$

where $\Lambda$ is the scale of the $S U\left(n_{c}\right)$ theory, because the unbroken $S U(r)$ interactions become strong at long distances. Of course, if (2.19) were not satisfied, semi-classical formulae like Eq.(2.12) would break down completely.

However, all is not lost. As long as the breaking scales $v_{0}, v_{j}$ are much larger than the scale of the $S U(r)$ theory, the properties of the stable states such as their multiplicity and charges as summarized in Table 2. should be correct. (Also, the mass formulae should be approximately valid.) They represent the nonzero elements of the homotopy group,

$$
\Pi_{2}\left(\frac{S U\left(n_{c}\right)}{S U(r) \times U(1)^{n_{c}-r}}\right)=\Pi_{1}\left(U(1)^{n_{c}-r}\right)=\mathbf{Z}^{n_{c}-r} .
$$

We assume that these properties are maintained as Higgs VEVS $v_{0}$ and $v_{j}$ 's are smoothly varied. Their masses will vary, of course, in an unknown way.

The central question is whether the unbroken $S U(r)$ gauge group is further dynamically broken by the strong $S U(r)$ interactions themselves. This depends on the system considered. In some model the $S U(r)$ symmetry may be broken further, e.g., to $U(1)^{r-1}$. In this case the " $r$-plet of non-Abelian monopoles" simply means the presence of $r$ approximately degenerate (as long as $v_{0}$, $v_{j}$ 's are all large compared to the scale of $S U(r)$ gauge theory) monopoles 7 . Only if $S U(r)$ is not dynamically broken further do these particles behave truely as the fundamental multiplet of the dual gauge group.

What is most remarkable in our opinion is the fact that this second option seems to be realized in the $r$-vacua of the softly broken $\mathcal{N}=2 S U\left(n_{c}\right)$ gauge theory [6]. Massless magnetic particles with precisely the properties listed in Table 2. appear in the fully quantum-mechanical low-energy effective action there. We propose that these are non-Abelian monopoles of the type discussed above.

It is also significant that, in the softly broken $\mathcal{N}=2 S U\left(n_{c}\right)$ theory, the $r$ vacua with a magnetic $S U(r)$ gauge group occur only for $r \leq \frac{n_{f}}{2}$. Before discussing this point further, we show in the next section that if fermions in the fundamental representation of the $S U\left(n_{c}\right)$ gauge group are introduced in the theory, each of them possesses one zero mode in the background of appropriate non-Abelian monopoles of the form, Eq.(2.8). It is then possible that the non-Abelian monopoles in the $\underline{r}$ of $S U(r)$ transform as a fundamental representation of the global $S U\left(n_{f}\right)$ symmetry. This explains the occurrence of non-Abelian monopoles carrying $S U\left(n_{f}\right)$ quantum numbers in the $r$-vacua of the softly broken $\mathcal{N}=2$ theory.

\footnotetext{
${ }^{2} N=2$ supersymmetric $S U\left(n_{c}\right)$ pure Yang-Mills theory is a good example of this types of theory. Indeed, in the case of $S U(3)$ theory, it can be seen that in an appropriate semi-classical domain of $u=\left\langle\operatorname{Tr} \Phi^{2}\right\rangle, v=\left\langle\operatorname{Tr} \Phi^{3}\right\rangle$, $\operatorname{such}$ that $a_{1}=-a_{2}$, there are two approximately degenerate monopoles. See Eq.(6.4) of Klemm et. al. [3].
} 


\section{Flavor Charges of Non-Abelian Nonopoles; Confinement versus Dy- namical Symmetry Breaking}

The mechanism with which the 't Hooft-Polyakov monopole acquires nontrivial flavor quantum numbers is well-known 11, 8, 12]. In order to show that an analogous result holds for our nonAbelian monopoles, consider a fermion $\psi_{L, R}$ in the fundamental representation ( $\left.\underline{3}\right)$ in the case of a $S U(3)$ theory broken as $S U(3) \rightarrow S U(2) \times U(1)$ considered above. $\psi_{L}$ and $\psi_{R}$ can be decomposed into $S U(2)_{u}$ multiplets:

$$
\psi_{L}=\psi_{L(2)} \oplus \psi_{L(0)}, \quad \psi_{R}=\psi_{R(2)} \oplus \psi_{R(0)}
$$

The zero-energy Dirac equations have the form 3

$$
\begin{gathered}
-\vec{\sigma} \cdot \vec{p} \psi_{L(2)}-e \vec{\sigma} \cdot(\vec{t} \wedge \hat{r}) A(r) \psi_{L(2)}-\frac{1}{2} v \psi_{R(2)}+3 v \vec{t} \cdot \hat{r} \psi_{R(2)} \phi(r)=0, \\
-\vec{\sigma} \cdot \vec{p} \psi_{L(0)}+v \psi_{R(0)}=0, \\
\vec{\sigma} \cdot \vec{p} \psi_{R(2)}+e \vec{\sigma} \cdot(\vec{t} \wedge \hat{r}) A(r) \psi_{R(2)}-\frac{1}{2} v \psi_{L(2)}+3 v \vec{t} \cdot \hat{r} \psi_{L(2)} \phi(r)=0, \\
\vec{\sigma} \cdot \vec{p} \psi_{R(0)}+v \psi_{L(0)}=0,
\end{gathered}
$$

where $\vec{t}=\left(t_{4}, t_{5}, \frac{t_{3}}{2}+\frac{\sqrt{3} t_{8}}{2}\right)$. These equations can be decoupled by using the combinations $\psi_{+}=$ $\psi_{L}+i \psi_{R}, \psi_{-}=\psi_{L}-i \psi_{R}$ as $\left(m \equiv \frac{v}{2}\right)$

$$
\begin{gathered}
-\vec{\sigma} \cdot \vec{p} \psi_{+(2)}-e \vec{\sigma} \cdot(\vec{t} \wedge \hat{r}) A(r) \psi_{+(2)}+i m \psi_{+(2)}-i 3 v \vec{t} \cdot \hat{r} \psi_{+(2)} \phi(r)=0, \\
-\vec{\sigma} \cdot \vec{p} \psi_{+(0)}-i v \psi_{+(0)}=0,
\end{gathered}
$$

and similarly for $\psi_{-(2)}$ and $\psi_{-(0)}$, with a minus sign in front of the corresponding terms with $m, v$ or $\phi(r)$. The singlet fermion obviously do not possess any zero modes. As for $\psi_{+(2)}$ and $\psi_{-(2)}$ Eq. (3.2) are formally the same as the equations for massive fermions with mass, $m \equiv \frac{v}{2}$. According to the Callias index analysis for massive fermions there is one normalizable zero mode if $|\phi|-|m|>0$; there are none otherwise [8, 12]. In our normalization, this condition reduces to

$$
\frac{3}{2} v>\frac{1}{2} v
$$

which is obviously satisfied. Therefore the doublet fermion possesses one zero mode.

An analogous construction in the case of the breaking $S U\left(n_{c}\right) \rightarrow S U(r) \times U(1)^{n_{c}-r}$, the above condition is replaced by

$$
\left|\frac{v_{0}-v_{r+1}}{2}\right|>\left|\frac{v_{0}+v_{r+1}}{2}\right| \text {. }
$$

Note that for the breaking $S U(n) \rightarrow S U(n-1) \times U(1)$ such a condition is always satisfied; otherwise, only the monopoles with VEVS satisfying the above condition will give rise to fermion zero modes.

3 The gamma matrices used by Jackiw and Rebbi, $\gamma_{J R}^{0}=\left(\begin{array}{cc}\mathbf{0} & -i \mathbf{1} \\ i \mathbf{1} & \mathbf{0}\end{array}\right) ; \gamma_{J R}^{i}=\left(\begin{array}{cc}-i \sigma_{i} & \mathbf{0} \\ \mathbf{0} & i \sigma_{i}\end{array}\right)$ and $\gamma_{c h}^{0}=$ $\left(\begin{array}{ll}\mathbf{0} & \mathbf{1} \\ \mathbf{1} & \mathbf{0}\end{array}\right) ; \quad \gamma_{c h}^{i}=\left(\begin{array}{cc}\mathbf{0} & \sigma_{i} \\ -\sigma_{i} & \mathbf{0}\end{array}\right)$ in the chiral representation used here, are related by $S \gamma_{J R}^{\mu} S^{-1}=\gamma_{c h}^{\mu}$, where $S=\frac{1}{2}\left(\begin{array}{cc}(1+i) \mathbf{1} & -(1+i) \mathbf{1} \\ (1-i) \mathbf{1} & (1-i) \mathbf{1}\end{array}\right)$. The Yukawa coupling has been set to unity. 
To construct explicitly the zero mode, we set

$$
\begin{aligned}
& \psi_{+(2)}=-i \sigma_{\alpha i}^{2} g_{+}(r)+\left(\vec{\sigma} \sigma^{2}\right)_{\alpha i} \hat{r} f_{+}(r), \\
& \psi_{-(2)}=-i \sigma_{\alpha i}^{2} g_{-}(r)+\left(\vec{\sigma} \sigma^{2}\right)_{\alpha i} \hat{r} f_{-}(r)
\end{aligned}
$$

in Eq.(3.3) and analogous one for $\psi_{-(2)}$. The scalar functions $g_{+}, f_{+}$satisfy coupled linear differential equations,

$$
g_{+}^{\prime}(r)+G_{+} g_{+}(r)+m f_{+}(r)=0, \quad f_{+}^{\prime}(r)+F_{+} f_{+}(r)+m g_{+}(r)=0,
$$

and similarly $\left(g_{-}, f_{-}\right)$with $(-)$in front of the mass terms. The functions appearing in the coefficients are $G_{+}(r)=\frac{3}{2} v \phi(r)+e A(r), G_{-}(r)=-\frac{3}{2} v \phi(r)+e A(r), F_{+}(r)=\frac{3}{2} v \phi(r)-e A(r)+\frac{2}{r}, F_{-}(r)=$ $-\frac{3}{2} v \phi(r)-e A(r)+\frac{2}{r}$. By eliminating $g_{+}$in favor of $f_{+}$(or vice versa) we get a second order differential equation involving $f_{+}$only (or $g_{+}$only). From the asymptotic behavior of the solutions of these equations we find that if the condition (3.4) is satisfied, there is one solution $\left(g_{+}, f_{+}\right)$regular at the origin, which is normalizable. Both solutions $\left(g_{-}, f_{-}\right)$are instead non-normalizable.

In the case of a $n_{f}$-flavored model, then, each fermion has one zero mode, in the background of each monopole. Denoting the quantized fermion field as $\psi_{i}=b_{i} \psi^{(0)}+\ldots, \quad\left(i=1,2, \ldots, n_{f}\right)$, where $b_{i}$ 's are the zero mode annihilation operator, the standard procedure yields the possible monopole multiplets,

$$
|k\rangle, \quad b_{i}^{\dagger}|k\rangle, \quad b_{i}^{\dagger} b_{j}^{\dagger}|k\rangle, \quad \ldots, \quad\left(k=1,2, \ldots, r ; i, j=1,2, \ldots, n_{f}\right)
$$

which belong to various antisymmetric irreducible representations of the flavor $S U\left(n_{f}\right)$ group $(\mathbf{1}$, $\square$, 日, etc.) When full quantum effects are taken into account, only the members of a given multiplet remain degenerate. It is a dynamical question which of them eventually become light in the infrared.

In the softly broken $\mathcal{N}=2 S U\left(n_{c}\right)$ theory with $n_{f}$ flavors, it turns out that the massless nonAbelian monopoles appearing in the $r$-vacua $(q, \tilde{q})$ carry a flavor quantum number of the fundamental representation ( $\square$ ) of the original flavor $S U\left(n_{f}\right)$ global symmetry group. This can now be understood as due to the Jackiw-Rebbi mechanism generalized to non-Abelian monopoles, (3.8).

The way their condensates break spontaneously the global $S U\left(n_{f}\right) \times U(1)$ symmetry in this theory is quite interesting. Upon $\mathcal{N}=1$ perturbation (adjoint mass term $\mu \Phi^{2}$ ), condensates of the form

$$
\left\langle q_{\alpha}^{i}\right\rangle=\delta_{\alpha}^{i} V, \quad\left\langle\tilde{q}_{\alpha}^{i}\right\rangle=\delta_{\alpha}^{i} V^{\prime}, \quad i, \alpha=1,2, \ldots, r
$$

where $V, V^{\prime} \propto \sqrt{\mu \Lambda}$, develop $[6$. On the one hand, they induce the (dual) Higgs mechanism, completely breaking the dual local $S U(r)$ group (confinement); at the same time they trigger dynamical symmetry breaking

$$
S U\left(n_{f}\right) \times U(1) \Longrightarrow U(r) \times U\left(n_{f}-r\right) .
$$

The way the condensate of non-Abelian monopoles break both (dual) color and flavor symmetries leaving the diagonal subgroup unbroken (Color Flavor Locking), shows an intriguing similarity to what is thought to happen in QCD at high quark densities [15]

\footnotetext{
${ }^{4}$ In the softly broken $\mathcal{N}=2 S U\left(n_{c}\right)$ theory under consideration here, the Yukawa coupling breaks the chiral $S U_{L}\left(n_{f}\right) \times S U_{R}\left(n_{f}\right)$ symmetry to the diagonal $S U\left(n_{f}\right)$ at the classical level, so the symmetry breaking pattern is peculiar to this model. Another difference is that in the high density QCD it is diquark composites that condense.
} 


\section{Duality}

That the $r$-vacua with effective, non-Abelian $S U(r) \times U(1)^{n_{c}-r}$ gauge symmetry exist only for $r<\frac{n_{f}}{2}$, thus only in theories with flavor, is a manifestation of the fact that the quantum behavior of non-Abelian monopoles depends crucially on the massless matter fermion degrees of freedom in the fundamental theory. Indeed, the magnetic $S U(r) \times U(1)^{n_{c}-r}$ theory with these matter multiplets is infrared-free (i.e., non asymptotic free). This is the correct behavior as it should be dual to the original asymptotic free $S U\left(n_{c}\right)$ gauge theory. Note that the gauge coupling constant evolution, which appears as due to the perturbative loops of magnetic monopoles, is actually the result of, and equivalent to, the infinite sum of instanton contributions in the original $S U\left(n_{c}\right)$ theory.

This is perfectly analogous to the observation [13] about how the old paradox related to the Dirac quantization condition and renormalization group [14] :

$$
g_{e}(\mu) \cdot g_{m}(\mu)=2 \pi n, \quad \forall \mu,
$$

is solved within the $S U(2)$ Seiberg-Witten theory.

This reasoning also leads to the explanation why in the pure $\mathcal{N}=2 S U\left(n_{c}\right)$ theory or on a generic point of the Coulomb branch of the $\mathcal{N}=2 \mathrm{SQCD}$, the low-energy effective theory is an Abelian gauge theory [1]-[3]. Massless fermion flavors are needed in order for non-Abelian monopoles to get dressed, via a generalized Jackiw-Rebbi mechanism discussed in Section 3. with a non trivial $S U\left(n_{f}\right)$ flavor quantum numbers and, as a result, to render the dual gauge interactions infrared-free. When this is not possible, non-Abelian monopoles are strongly coupled and do not manifest themselves as identifiable low-energy degrees of freedom.

In this respect, it is very interesting that the boundary case $r=\frac{n_{f}}{2}$ also occurs (confining vacua of type (iii) discussed in Introduction) within the class of supersymmetric theories considered in [6]. In these vacua, non-Abelian monopoles and dyons are strongly coupled, but still describes the low-energy dynamics, albeit via non-local effective interactions. The situation is somewhat similar to what happens in the $\mathcal{N}=4$ theories [17].

\section{Summary and Discussion}

Non-Abelian monopoles are elusive objects. Though their presence may be detected in a semiclassical approximation, their true nature depends on the long distance physics. If the unbroken gauge group is dynamically broken further in the infrared such multiplets of states simply represent an approximately degenerate set of magnetic monopoles. Only if there is no further dynamical breaking do the non-Abelian monopoles transforming as nontrivial multiplets of the unbroken, dual gauge group, appear in the theory.

We have shown that there are strong indications that this occurs in the $r$-vacua (with an effective $S U(r) \times U(1)^{n_{c}-r}$ gauge symmetry) of the softly broken $\mathcal{N}=2, S U\left(n_{c}\right)$ supersymmetric QCD [6]. If our idea is correct, this is perhaps the first physical system known in which Goddard-NuytsOlive-Weinberg monopoles manifest themselves as infrared degrees of freedom, playing an essential dynamical role.

More direct verification of the above relation, such as done in the cases of Abelian monopoles [18], seems to be difficult however, for the subtleties discussed earlier. 
Our arguments apply equally well to the softly broken $\mathcal{N}=2$ theories with $U S p\left(2 n_{c}\right)$ or $S O\left(n_{c}\right)$ gauge groups, with nonvanishing bare (equal) quark masses. Semiclassically these gauge groups can be broken to $S U(r) \times U(1)^{n_{c}-r+1}$, and non-Abelian monopoles appearing there can be identified with the dual quarks in the low-energy magnetic $S U(r) \times U(1)^{n_{c}-r+1}$ theory.

Our observation is consistent with an interpretation suggested in [6] (see also [16]), that these magnetic quarks might be regarded as baryonic constituents of certain $U(1)$ monopole,

$$
U(1) \text { monopole } \sim \epsilon^{a_{1} \ldots a_{r}} q_{a_{1}}^{i_{1}} q_{a_{2}}^{i_{2}} \ldots q_{a_{r}}^{i_{r}}
$$

As the dual $S U(r)$ interactions are infrared-free, the Abelian monopole breaks up into its constituents. The non-Abelian monopoles carry a minimum $\mathbf{Z}_{r}$ charge: its $U(1)$ charge is $\frac{1}{r}$ with respect to that of an Abelian monopole (for $U(1)$ lying within the $S U(r)$ subgroup), in accordance with the above.

Another way to relate our massless non-Abelian monopoles to something known, would be to consider, within the softly broken $\mathcal{N}=2$ theories, the large and equal bare quark mass regime. Upon addition of the $\mathcal{N}=1$ perturbation, classical supersymmetric vacua exist with $S U(r) \times S U\left(n_{c}-r\right)$ $\times U(1)$ gauge symmetry unbroken and with $n_{f}$ massless quarks in the $\underline{r}$ of $S U(r)$ [6]. As this group

is infrared free for $r<\frac{n_{f}}{2}$, the physics at low energies is described by the classical Lagrangian. As $m$ is varied and reaches values below the dynamical scale $\Lambda$ of the theory, however, the change of monodromy around the quark singularity occurs, when it moves below the cuts produced by other singularities. Quarks become magnetic monopoles [2]. Precise way this type of mehamorphosis takes place has been studied explicitly only in the simplest cases of $S U(2)$ theories [19] (see [20] for some results in $S U(3)$ pure Yang Mills theory) with Abelian monopoles only. Extension of these studies to non-Abelian monopoles seem to be challenging.

In view of the many known examples of $\mathcal{N}=1$ Seiberg's duality 21 in which dual magnetic monopoles with non-Abelian charges play prominent roles, what is the significance of the present work? The fact is that in spite of overwhelming indirect evidences that duality features discussed in [21] are indeed correct, the detailed understanding of the magnetic variables appearing in these models is still lacking. For instance, an attempt to "understand" the origin of such a duality starting from the softly broken $\mathcal{N}=2$ theory, had only a partial success $[5]$. Any further steps, such as the ones taken here, towards deepening our insight into the nature of non-Abelian magnetic monopoles, should be welcome.

\section{Acknowledgments}

The authors thank Roberto Auzzi, Roberto Grena, Jarah Evslin, Adam Ritz, Dave Tong, Alyosha Yung and Valya Zakharov for stimulating discussions as well as for useful informations. One of the authors (K.K.) thanks the organizers and participants of the Workshop "Continuous Advances in QCD", ArkadyFest, Minneapolis, May, 2002, in which some of the ideas in this work have been presented, for a pleasant atmosphere and for discussions.

\section{References}


[1] N. Seiberg and E. Witten, Nucl.Phys. B426 (1994) 19, hep-th/9407087; Erratum ibid. Nucl.Phys. B430 (1994) 485.

[2] N. Seiberg and E. Witten, Nucl. Phys. B431 (1994) 484, hep-th/9408099.

[3] P. C. Argyres and A. F. Faraggi, Phys. Rev. Lett 74 (1995) 3931, hep-th/9411047; A. Klemm, W. Lerche, S. Theisen and S. Yankielowicz, Phys. Lett. B344 (1995) 169, hepth/9411048; Int. J. Mod. Phys. A11 (1996) 1929, hep-th/9505150; A. Hanany and Y. Oz, Nucl. Phys. B452 (1995) 283, hep-th/9505075; P. C. Argyres, M. R. Plesser and A. D. Shapere, Phys. Rev. Lett. 75 (1995) 1699, hep-th/9505100; P. C. Argyres and A. D. Shapere, Nucl. Phys. B461 (1996) 437, hep-th/9509175; A. Hanany, Nucl.Phys. B466 (1996) 85, hep-th/9509176.

[4] G. 't Hooft, Nucl. Phys. B190 (1981) 455. S. Mandelstam, Phys. Lett. 53B (1975) 476; Phys. Rep. 23C (1976) 245.

[5] P. C. Argyres, M. R. Plesser and N. Seiberg, Nucl. Phys. B471 (1996) 159, hep-th/9603042; P.C. Argyres, M.R. Plesser, and A.D. Shapere, Nucl. Phys. B483 (1997) 172, hep-th/9608129.

[6] G. Carlino, K. Konishi and H. Murayama, JHEP 0002 (2000) 004, hep-th/0001036; Nucl.Phys. B590 (2000) 37, hep-th/0005076, K. Konishi, Proceedings of Continuous Advances in QCD, Minneapolis, Minnesota, May 2000, hep-th/0006086; G. Carlino, K. Konishi, Prem Kumar and H. Murayama, Nucl.Phys. B608 (2001) 51, hep-th/0104064.

[7] P. Goddard, J. Nuyts and D. Olive, Nucl. Phys. B125 (1977) 1.

[8] E. Weinberg, Nucl. Phys. B167 (1980) 500; Nucl. Phys. B203 (1982) 445.

[9] G. 't Hooft, Nucl. Phys. B79 (1974) 276; A.M. Polyakov, JETP Lett. 20 (1974) 194.

[10] A. Abouelsaood, Nucl. Phys. B226 (1983) 309; P. Nelson and A. Manohar, Phys. Rev. Lett. 50 (1983) 943; A. Balachandran et. al., Phys. Rev. Lett. 50 (1983) 1553; P. Nelson and S. Coleman, Nucl. Phys. B227 (1984) 1; E. Weinberg, Phys.Rev.D54 (1996) 6351, hep-th/9605229.

[11] R. Jackiw and C. Rebbi, Phys. Rev. D13 (1976) 3398.

[12] C. Callias, Comm. Math, Phys. 62 (1978) 213; J. de Boer, K. Hori and Y. Oz, Nucl. Phys. B500 (1997) 163, hep-th/9703100.

[13] M. Di Pierro and K. Konishi, Phys.Lett.B388 (1996) 90, hep-th/9605178.

[14] B. Zumino, Erice Lectures (1966), Ed. A. Zichichi; S. Coleman, Erice Lectures (1977), Ed. A. Zichichi.

[15] M. G. Alford, K. Rajagopal and F. Wilczek, Nucl.Phys. B537 (1999) 443, hep-ph/9804403.

[16] J. Evslin, H. Murayama, U. Varadarajan and J. E. Wang, JHEP 0111 (2001) 030, hepth/0107072.

[17] C. Montonen and D. Olive, Phys. Lett. 72B (1977) 117.

[18] K. Konishi and H. Terao, Nucl.Phys.B511 (1998) 264, hep-th/9707005. 
[19] A. Bilal, F. Ferrari, Nucl.Phys.B516 (1998) 175, hep-th/9706145; A. Cappelli, P. Valtancoli and L. Vergnano, Nucl.Phys.B524 (1998) 469, hep-th/9710248.

[20] B. J. Taylor, JHEP 0108 (2001) 031.

[21] N. Seiberg, Nucl.Phys.B435 (1995) 129, hep-th/9411149; D. Kutasov, A. Schwimmer and N. Seiberg, Nucl.Phys.B459 (1996) 455, hep-th/9510222. 